\begin{tabular}{|c|c|}
\hline \multirow{3}{*}{ 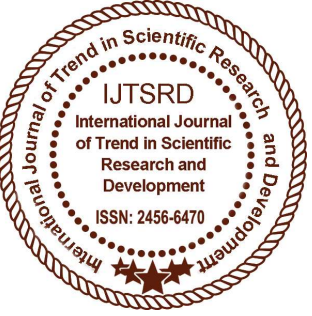 } & $\begin{array}{l}\text { International Journal of Trend in Scientific } \\
\text { Research and Development (IJTSRD) }\end{array}$ \\
\hline & International Open Access Journal \\
\hline & ISSN No: 2456 - 6470 | www.ijtsrd.com | Volume - 2 | Issue - 5 \\
\hline
\end{tabular}

\title{
Effect of Ion Refocusing and Focusing at the Ne and Ar Small Angle Ion Bombardment on the Surface III-V Compound Semiconductors
}

\author{
Karimov Muxtor Karimberganovich \\ Department of Physics, Faculty of Physic and \\ Mathematics of Urgench State University, \\ Urgench, Uzbekistan
}

\author{
Sobirov Ravshanbek Yuldashbaevich \\ Magistr of Department of Physics, Faculty of Physic \\ and Mathematics of Urgench State University, \\ Urgench, Uzbekistan
}

\section{Sadullaev Shuxrat Ravshanovich \\ Teacher at the Academium Lyceum at the Urgench Branch of TUIT,} Urgench, Uzbekistan

\begin{abstract}
In this paper presents small angle ion scattering of noble gases from the III-V compound semiconductor surfaces have been studied by the method of computer simulation. The effect ion focusing and refocusing was studied. The coefficient of scattering ions has been calculated.
\end{abstract}

Keywords: ion focusing; ion refocusing; computer simulation; ion scattering; semi channels;

\section{INTRODUCTION}

Indium phosphide (InP) is an important III-V semiconductor, it exists in crystalline form zinc blende (ZB) with direct band gaps of 1.42 and $1.35 \mathrm{eV}$ at room temperature, respectively, and is a highly promising candidate for construction of viable nano-integrated circuits [1-3]. The low dimensions of InP in the radial direction exhibit size confinement effects that give novel physical properties to low-sized InP as compared to bulk materials.

InP semiconductors among other III-V semiconductors are used in high power and highfrequency electronics because of high electron velocity when compared with other III-V semiconductors, for instance, GaAs. Moreover, InP has the longest lived optical phonons among the compounds with ZB crystal structure [4,5]. Therefore surface structure InP very interesting as a optoelectronic device.

At the study of surface InP are used a lot of methods. One of them is a method of low energy ion scattering spectroscopy (LEIS). LEIS probes the elemental composition of the outermost atomic layer of a material and provides static depth profiles of the outer $c a .10 \mathrm{~nm}$ of surfaces. Its extreme surface sensitivity and quantitative nature make it a powerful tool for studying the relationships between surface chemistry and surface related phenomena such as wetting, adhesion, contamination, and thin film growth. The high depth resolution obtained in LEIS in its static and sputter depth profile modes are useful for studying the layer structures of thin films. LEIS instrumentation has improved significantly in recent years, showing dramatic increases in its sensitivity and further expanding its potential applications [6-8].

The extraordinary surface sensitivity of low energy ion scattering spectrometry may be applied to the characterization of a wide variety of materials. On the one hand, the surface chemistry may be determined, using ion scattering spectrometry for engineering materials used in diverse technologies such as lubrication and adhesive bonding; such surface chemistry, perhaps involving only a few monolayer's, may govern the success or failure of a process. On the other hand, ion scattering spectrometry is also useful 
in investigating the chemistry and physical and electronic structure of single crystals.

In this article, we provide a practical introduction to the technique, including a discussion of the basic theory of LEIS, ion focusing and refocusing effects. The main our goal was study of ion focusing and refocusing effects which observed in surface semichannels.

\section{Computational method and discussion}

The collision between particles is commonly described by using two coordinate system: the laboratory system and the centre of mass system. The laboratory system of coordinates (L-system) is a system in which the scattering particles is at rest before collision The centre of mass system (CMsystem) is a system in which the centre of mass of the particles is a rest both before and after collision. Figure 1 presents the scattering snapshot in the Lsystem. A particle of mass $\mathrm{M}_{1}$ strikes with a velocity $\mathrm{v} 0$ the target atom which is initial at rest and whose mass $\mathrm{M}_{2}$. If there were no interaction between the incidence particle and the target atom, the particle would pass at a distance $\mathrm{p}$ from the target atom. This distance is called the impact parameter of the collision. As a result of the interaction the particle is scattered through an angle $\Theta_{1}$ relative to the direction of its initial motion. As regard the target atom, after the collision it moves in a direction that makes an angle $\Theta_{2}$ with the direction of the initial motion of the incidence particle. This atom called a recoil atom. In this figure also illustrated the trajectory of motion of CM system. If the collision is elastic, i.e., if it not accompanied by a charge in the internal state of the particles, then from the laws of conservation kinetic energy and momentum we obtain expressions both for the energy of the particle scattered through an angle $\Theta_{1}$

$$
E_{1}=E_{0}\left(\frac{M_{1}}{M_{1}+M_{2}}\right)^{2}\left\{\left(\cos \theta_{1} \pm\left(\frac{M_{2}}{M_{1}}\right)^{2}-\sin 2 \theta 1\right)^{1 / 2}\right\}^{2}
$$

If the mass of the incidence particle is smaller than the mass of the target atom, the plus sign in above formula. If $\mathrm{M}_{1}>\mathrm{M}_{2}$, both sign are taken before the square root in above mention formula,
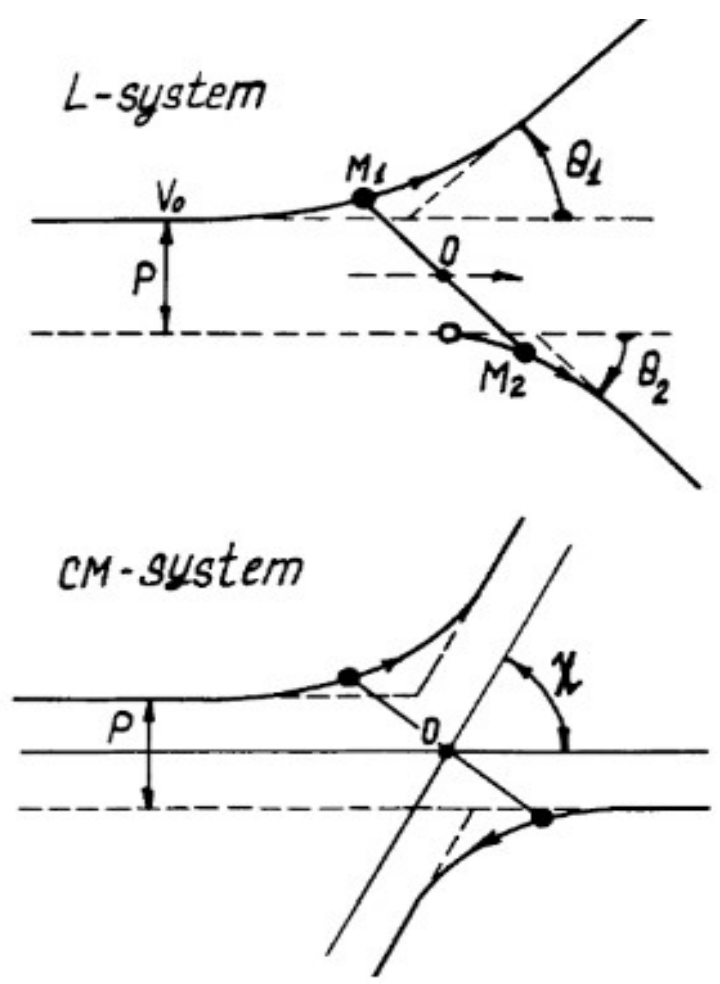

Figure.1: Scattering geometry for two colliding particles in the laboratory and in the centre of mass system.

The numerical code used in this work is based on the consequence binary collision approximation [9]. Using the universal potential of Ziegler-BiersackLittmark interaction [10] and accounting for time integral the trajectories of ions testing for grazing scattering were simulated on discrete row of atoms and on semi channels on a single crystal surface. For consideration of possible simultaneous collisions of ion with several target atoms, the procedure proposed by Robinson and Torrens [11] was used. The simulations were run with the crystal atoms initially stationary at equilibrium lattice sites because in the conditions of grazing incidence the influence of the thermal vibrations of lattice atoms at room temperature on ion scattering results is insignificant. The elastic and inelastic losses of energy have been summed along the trajectory of scattered ions. Inelastic losses of energy were calculated by modified Firsov formula [12] and included into the scattering kinematics. The incident ions were followed throughout their slowing-down process until their energy falls below a predetermined energy of $25 \mathrm{eV}$.

Investigations of the processes of ion scattering on a single crystal at small angle incidence shown that there are effects, the presence of which is explained by the model of the semi channels formed on the surface of solids. 
We have studied the processes of scattering of $\mathrm{Ne}^{+}$ and $\mathrm{Ar}^{+}$ions from the surfaces of $\mathrm{InP}(001)<110>$ single crystals, for small values of the angle incidence with the initial energy of the incident particles equal to $\mathrm{E}_{0}=3$ and $5 \mathrm{keV}$. As a result, the dependence of the ion scattering coefficient on the angle of incidence was obtained.

It should be noted that the penetration of ions into the inside of the semi channel depends on its shape and size. It is known that at $\psi=0^{0}$, ions are scattered from atomic chains formed on the surface layers. With an increase in the angle of incidence, the ions can move in semichannels with different geometric parameters, depending on the direction of the single crystals.

Figure 2 shows the dependence of the scattering coefficient (Kp) of $\mathrm{Ne}^{+}$(black line) and $\mathrm{Ar}^{+}$(red line) ions from the InP (100) surface in the $<110>$ directions with $\mathrm{E}_{0}=5 \mathrm{keV}$ from the angle of incidence. It is clearly seen from the dependence that this dependence has a two-peak structure, the first intense peak referring to refocused ions on the surface semichannels. This effect is manifested in the fact that the ions begin to penetrate into the semichannel and are scattered from the atoms of this semichannel. These refocused ions can not penetrate into the neighbor semichannel, since the values of the angle of the incidence are very small.

This dependence shows that the $\mathrm{Ne}^{+}$ions begin to penetrate into the semichannel at $\psi=0,76^{0}$ and $\mathrm{Ar}^{+}$ ions start penetrate $\psi=0,85^{\circ}$. These results shows that the increasing of atom mass incidence ions increased angle of refocusing.

Our calculations showed that the values of the angles of incidence at which the ions begin to penetrate into the semicircle mainly depend on its geometric parameters (the width of the half-channel and the distance between the atoms) and the types of atoms that are on the surface layers. By the increasing angle of incidence the value of coefficient scattering ions decreased. This effect explained by influence of lower channels of surface semichannel.

On the dependence also we can observe two peaks on $\psi=2,15^{0}\left(\right.$ for $\mathrm{Ne}^{+}$) and $\psi=2,55^{0}$ (for $\mathrm{Ar}^{+}$).

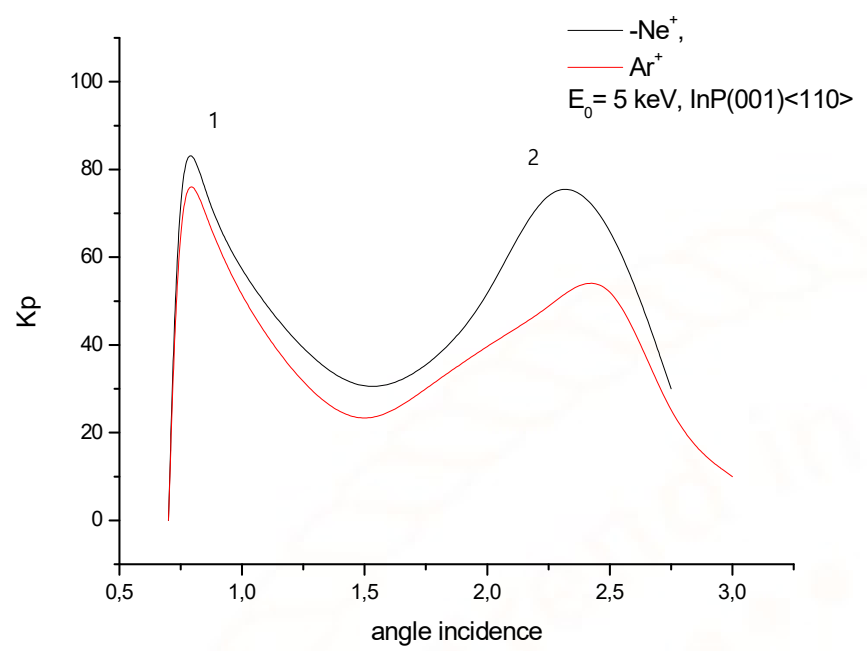

Figure 2. Dependence of the scattering coefficient (Kp) of $\mathrm{Ne}^{+}$(black line) and $\mathrm{Ar}^{+}$(red line) ions from the $\operatorname{InP}(100)$ surface in the $<110>$ directions with $\mathrm{E}_{0}$ $=5 \mathrm{keV}$ from the angle of incidence.

At these values of the angle of incidence, the ion focusing effect is observed, which manifests itself in the growth of the intensity and the decrease in the half-width of the spatial distribution of the scattered flux for certain target orientations with respect to the incident beam. This effect consists in the fact that the ions passing between two atomic rows of the surface are focused on the bottom of the semichannel.

On the figure 3 presents the dependence $\mathrm{Kp}(\psi)$ for $\mathrm{Ne}^{+}$(black line) and $\mathrm{Ar}^{+}$(red line) scattered ions from the $\operatorname{InP}(001)<110>$ surface at the $\mathrm{E}_{0}=3 \mathrm{keV}$.

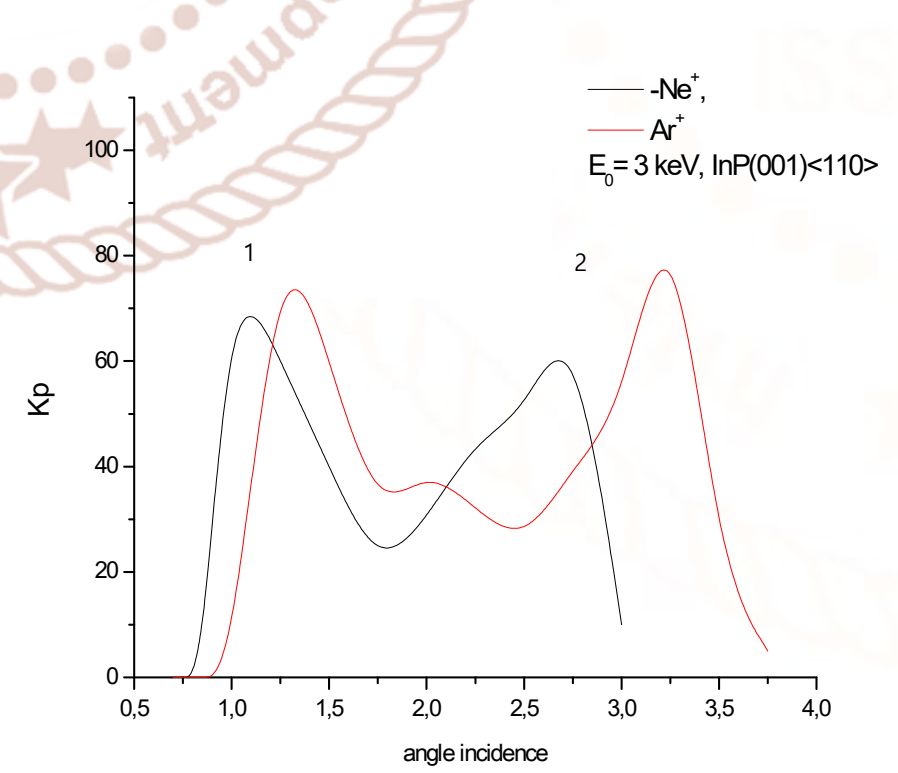

Figure 3. Dependence $\mathrm{Kp}(\psi)$ for $\mathrm{Ne}^{+}$(black line) and $\mathrm{Ar}^{+}$(red line) scattered ions from the $\operatorname{InP}(001)<110>$ surface at the $\mathrm{E}_{0}=3 \mathrm{keV}$. 
As well as in the previous case, this dependence has two intensive peaks: peak refocusing ions, and peak focusing ions. It is necessary to note, that these two dependences have essential distinctions, which are, that in case of $\mathrm{Ne}^{+}$and $\mathrm{Ar}^{+}$ions the peaks refocusing ions, and peak focusing ions have the large angles of incidence in comparison with a $\mathrm{E}_{0}=5 \mathrm{keV}$.

\section{Conclusions}

The dependences of the ion scattering coefficient for the surface of InP (100) single crystal on the angle of incidence are calculated. It is shown that the dependence of the ion scattering coefficient on the angle of incidence has a two-peak structure, and between these two intense peaks there are decreases in the scattering coefficient, which can be explained by the large number of reflected particles at large scattering angles and the localization of ions in the crystal.

\section{Acknowledgement}

The data used here to illustrate the image processing were recorded during calculation funded by the Agence Science and Technology Republic of Uzbekistan (Grants No.OT-F2-65).

\section{References}

1. Bjork M. T, Ohlsson B. J, Thelander C, Persson A. I, Deppert K, Wallenberg L. R, Samuelson L. Nanowire resonant tunneling diodes// Appl. Phys. Lett. $\quad 81, \quad 2002$ 2 4458-4460. (doi:10.1063/1.1527995).

2. Bjork MT, Ohlsson BJ, Sass T, Persson AI, Thelander C, Magnusson MH, Deppert K, Wallenberg LR, Samuelson L. 2002 Onedimensional heterostructures in semiconductor nanowhiskers. Appl. Phys. Lett. 80, 1058-1060. (doi:10.1063/1.1447312)

3. Fonseka HA, Caroff P, Wong-Leung J, Ameruddin AS, Tan HH, Jagadish C. 2002 Nanowires grown on InP (100): growth directions, facets, crystal structures, and relative yield control. ACS Nano 8, 6945-6954. (doi:10.1021/nn5017428)
4. U. Kutliev A. Dzhurakhalov, F. Umarov. Low energy ion scattering by atomic steps on the single crystal surface// Radiation Effects and Defects in Solids. Incorporating Plasma Science and Plasma Technology. Traylor Francis. 2002.T.159.N.5.P.293-299.

5. Karimov M. K, Narimonov N, Kutliev U. O. Study of Ion Scattering Process by the Method of Binary Collision Approximation// International Journal of Trend in Scientific Research and Development. 2017. T1. Issue-4.

6. Dzhurakhalov A., Kutliev U. O., Kalandarov B. S. Umarov F. F. Computer simulation of $\mathrm{Ne}$ ions scattering from the $\mathrm{Cu}(100)$ and $\mathrm{GaP}$ (100) surfaces at the grazing incidence//Uzbek Journal of Physics.-Tashkent.2007.-Vol.9.N5-6.P.317324.

7. Dzhurakhalov A. A., Kutliev U. O. Peculiarities of $\mathrm{Ne}+$ ion scattering from the GaP (100)surface at grazing incidence// Surface investigation.Singapore, 2000.-vol.15, -P. 705-710.

8. Dzhurakhalov A. A., Kalandarov B. S., Kutliev. U. O., Umarov F. F. The ion dechanneling mechanism at grazing scattering on the surface atomic steps// IEEE Publishing. 0-7803-6462-7// 00. -New York, 2000.-P.217-219.

9. Dzhurakhalov A. A., Kutliev U. O., Rasulov A. Atomic scale analysis of defect structures in single crystals by ion scattering and channeling.// Engineering Mechanics. Amsterdam, 2004, N5.Vol.11.-P. 1-4.

10. O'Connor D. J., Biersack J. P. Comparison of theoretical and empirical potentials //Nucl. Instr. Meth. Phys. Res. Amsterdam, 1986.-V.B15.P.1417.

11. Robinson M., Torrens L. Computer simulation of atomic displacement cascades in solids in the binary collision approximation//Phys.Rev.USA, 1974.- vol.B9.-P.5008-5024.

12. Dzhurakhalov A. A., Kutliev U.O. Peculiarities of $\mathrm{Ne}^{+}$ion scattering from the $\mathrm{GaP}(100)$ surface at grazing incidence//Surface investigation. Singapore, 2000.-vol.15, -P. 705-710. 